Revue d'histoire de l'Amérique française

REYUE D.HISTOIRE DE L'AMÉRIQUE FRANÇAISE

\title{
THÉRIAULT, Joseph Yvon, Anne GILBERT et Linda CARDINAL, dir., L'espace francophone en milieu minoritaire au Canada. Nouveaux enjeux, nouvelles mobilisations (Montréal, Fides, 2008), $562 \mathrm{p}$.
}

\section{François Charbonneau}

Volume 62, numéro 3-4, hiver-printemps 2009

La culture catholique

URI : https://id.erudit.org/iderudit/038540ar

DOI : https://doi.org/10.7202/038540ar

Aller au sommaire du numéro

Éditeur(s)

Institut d'histoire de l'Amérique française

ISSN

0035-2357 (imprimé)

1492-1383 (numérique)

Découvrir la revue

Citer ce compte rendu

Charbonneau, F. (2009). Compte rendu de [THÉRIAULT, Joseph Yvon, Anne GILBERT et Linda CARDINAL, dir., L'espace francophone en milieu minoritaire au Canada. Nouveaux enjeux, nouvelles mobilisations (Montréal, Fides, 2008), 562 p.] Revue d'histoire de l'Amérique française, 62(3-4), 604-610.

https://doi.org/10.7202/038540ar d'utilisation que vous pouvez consulter en ligne. 
prédécesseurs (Marcel Trudel, John A. Dickinson, Mathieu d'Avignon, entre autres) semblent inconnus de l'auteur, qui suppose que tous partagent son admiration: "Tous les historiens familiers avec la vie et les exploits de Champlain, catholiques ou protestants, Anglais, Français ou Néerlandais d'origine, citoyens anglais, canadiens, français ou des ÉtatsUnis d'Amérique, sont visiblement frappés des qualités et des vertus de Champlain» (ibid., p. 332-333)!

D'entrée de jeu, l'éditeur admet qu’à l'origine, le «manuscrit était dans une forme inachevée et assez déroutante» («Préface», ibid., p. 9). En considération des raisons susdites et pour plusieurs autres, il faut conclure que le livre demeure déroutant, induit trop souvent en erreur les lecteurs et ne respecte pas la méthodologie élémentaire de toute biographie historique. Il n'aurait pas dû être publié sous sa forme actuelle. Cette biographie ne recèle rien qui n'ait déjà été écrit par les historiens au fil des ans. Tout au plus les lecteurs y trouveront-ils une version augmentée du Samuel de Champlain de Joe C. W. Armstrong (Montréal, Éditions de l'Homme, 1988) - à la différence que Séguin n’identifie que très rarement ses sources et ses références (sauf dans les deux derniers chapitres) - à laquelle l'auteur a greffé quelques mises en contexte. Cet ouvrage sera tout de même "pertinent" pour toute étude des représentations de Champlain et de ses contemporains, n'étant qu'un livre ordinaire et fort décevant sur Champlain, édité au temps du $400^{\mathrm{e}}$ anniversaire de la fondation de Québec. Hélas, il nous ramènera «en arrière» dans le temps...

MATHIEU D’AVIGNON

Groupe de recherche sur l'histoire Université du Québec à Chicoutimi

THÉRIAULT, Joseph Yvon, Anne GILBERT et Linda CARDINAL, dir., L'espace francophone en milieu minoritaire au Canada. Nouveaux enjeux, nouvelles mobilisations (Montréal, Fides, 2008), $562 \mathrm{p}$.

Cet ouvrage collectif est en quelque sorte la suite du livre Francophonies minoritaires au Canada, l'état des lieux, paru en 1999. Alors que le premier ouvrage était davantage descriptif, les 12 textes que comporte ce livre offrent une réflexion poussée et ambitieuse sur les principaux enjeux auxquels font face les francophones du Canada vivant à l'extérieur du Québec. Disons-le franchement, le portrait brossé par les auteurs est plutôt sombre: malgré les importants gains réalisés par les francophones 
depuis l'adoption de la Charte canadienne des droits et libertés, la francophonie canadienne affronte toujours d'importants défis, qu'il s'agisse des transferts linguistiques, de l'acculturation ou encore du déficit démocratique.

Le coup d'envoi est donné par Anne Gilbert qui interroge de manière exhaustive le concept de vitalité des communautés francophones en situation minoritaire. L'auteure s'intéresse d'abord au concept, avant de déterminer si, effectivement, l'on peut attribuer ce qualificatif aux communautés francophones du Canada en situation minoritaire. Le concept de vitalité renvoie à la capacité des communautés de survivre et de s'épanouir. Tout en reconnaissant l'ambiguiité du concept, l'auteure montre de manière satisfaisante qu'un ensemble de critères (nombre et poids relatifs des locuteurs, engagement communautaire, nombre et dynamisme des acteurs et des institutions, et ainsi de suite) permet effectivement de jauger non pas quantitativement, mais de manière qualitative, de la vitalité d'un groupe en situation linguistique minoritaire. L'angle d'approche de Gilbert pour appréhender cette vitalité est de s'intéresser aux espaces (géographiques, bien sûr, mais aussi «relationnels", comme les réseaux) dans lesquels se déploie la francophonie. L'intérêt de cette contribution est double. Il s'agit d'abord d'une extraordinaire synthèse de l'ensemble des enjeux (et travaux de recherche) liés à la présence et à la pérennité des communautés linguistiques en situation minoritaire. Gilbert s'intéresse tout particulièrement au rapport complexe qui lie individu et communauté dans la pérennité linguistique de l'un comme de l'autre. Cette contribution est digne d'intérêt, ensuite, si on replace ce texte dans l'ensemble de l'œuvre intellectuelle de la géographe. Jamais ses conclusions n'auront en effet été aussi sévères: «les communautés francophones ne bénéficient plus aujourd'hui des conditions nécessaires pour achever l'espace que l'histoire leur a légué» (p. 9).

Dans une perspective sociolinguistique cette fois, Devault, Allard et Landry se penchent eux aussi sur la question de la vitalité. Ceux qui s'intéressent à la francophonie en situation minoritaire connaîtront déjà les principales conclusions de ce texte puisque ces auteurs sont devenus au fil du temps une référence dans l'étude des comportements langagiers au Canada. Le lecteur qui ne connaît pas ces travaux y découvrira une bonne synthèse. Les autres n'y apprendront rien de bien nouveau, si ce n'est qu'ils constateront que les auteurs en sont maintenant à l'étape du fine tuning, avec l'introduction de concepts (vécu enculturant, vécu autonomisant, etc.) qui visent à préciser davantage la manière et les processus 
qui président à la formation de l'identité ethnolinguistique. La principale thèse des auteurs est que le comportement langagier d'un individu dépend largement de sa socialisation (pourcentage de membres du groupe endogame vivant dans la même région, complétude institutionnelle, et ainsi de suite), mais que cette socialisation n'est pas un déterminisme puisque l'individu "est aussi le produit d'une construction autonome et personnelle» (p. 74). Si les auteurs, grands statisticiens devant l'Éternel, sont tout à fait convaincants en ce qui a trait au processus de socialisation, la démonstration des phénomènes relevant de la construction autonome et personnelle est plus ardue. Le problème ne réside évidemment pas au niveau de l'analyse que proposent les auteurs, souvent intuitivement convaincante, mais de la difficulté de la démonstration elle-même, beaucoup plus ardue à ce niveau d'analyse microsociale. En d'autres termes, une étude macrosociale montre assez facilement que la langue minoritaire se porte bien là où les francophones sont concentrés. Il est beaucoup plus difficile de comprendre pourquoi, au niveau cette fois de l'individu, un type particulier de comportement langagier est adopté. La démonstration des auteurs s'accompagne ainsi de modèles graphiques... impressionnants.

Tous le textes qui suivent ces deux premiers retiennent un thème particulier. Celui de Diane Farmer s'intéresse au phénomène de l’immigration en contexte linguistique minoritaire. L'auteure a saisi le principal enjeu de cette nouvelle donne. L'immigration est vue par les communautés francophones en situation minoritaire comme une solution, au moins partielle, au problème de la baisse de leur poids démographique. Mais les communautés francophones en situation minoritaire, étant sans frontière géographique, se sont toujours définies «à partir de frontières linguistiques et culturelles» (p. 122). La tentative d'intégrer les immigrants à ces communautés force à une redéfinition de ces frontières. Si ce texte pose les bonnes questions et offre une réflexion de haut niveau sur les enjeux, on peut cependant reprocher à l'auteure d'avaliser sans distance critique les analyses que proposent du dossier certains commentateurs autorisés. Pour ne prendre qu'un exemple, l'auteur cite la commissaire aux langues officielles pour affirmer avec elle que «Citoyenneté et Immigration Canada s'est montré particulièrement actif dans la promotion des nouvelles dispositions liées à l'immigration en milieu minoritaire» (p. 125). Quiconque a eu l'occasion de transiger avec Citoyenneté et Immigration Canada sur la question de l'immigration au Canada trouverait une telle déclaration, au mieux, surprenante. 
Le texte d'André Leclerc s'intéresse à la question des enjeux économiques de la francophonie canadienne et offre un tableau peu reluisant des succès économiques de la francophonie canadienne. Bien que par comparaison avec les anglophones du pays, les retards économiques soient largement résorbés par rapport à l'époque de la commission LaurendeauDunton, il ne semble pas exister (hormis dans les territoires du NordOuest), de prime au bilinguisme. Au contraire et sans qu'il n'y ait de phénomène de cause à effet, ceux qui abandonnent le français à la maison réussissent beaucoup mieux économiquement que ceux qui ne parlent que le français, les personnes bilingues ne sont pas avantagées lors de l'embauche de cadres dans l'entreprise privée, etc. Enfin, malgré les importants investissements et les nombreux organismes voués à l'amélioration économique des francophones, les résultats semblent se matérialiser difficilement dans la réalité, nous apprend André Leclerc.

De leur côté, Annie Pilote et Marie-Odile Magnan proposent une réflexion sur l'école en milieu minoritaire qui, on le sait, a connu une fulgurante progression depuis l'adoption de la charte des droits et libertés. Après un important survol des nouveaux enjeux liés au rôle de ces institutions dans la construction identitaire (notamment celui de l'immigration ou encore du type de pédagogie à privilégier), les auteures en appellent à une réflexion sur le rôle de l'école dans la transmission de l'identité francophone. Louise Bouchard et Anne Leis signent un texte sur le développement de réseaux de santé en langue française, réseaux nés dans la foulée de la crise de l'Hôpital Montfort en 1998. Lucie Hotte offre quant à elle une excellente contribution sur la création en milieu minoritaire. Elle expose de brillante façon le conflit qui déchire le créateur en milieu minoritaire, tiraillé qu'il est entre le désir esthétique de laisser porter son œuvre par la créativité pure, et la quasi-obligation qui le ramène invariablement à «engager [son] art dans la promotion, la valorisation et la défense du groupe» (p. 319).

Trois textes intéresseront peut-être davantage les historiens. Il y a d'abord celui de Michel Bock, qui porte sur la question de la mémoire du Canada français. L'auteur montre la complexité du rapport à la mémoire du Canada français, tant au Québec que dans la francophonie canadienne et procède, pour ce faire, à un vaste et passionnant récapitulatif des débats et contributions ayant porté sur cet enjeu. Bock met bien la table à JosephYvon Thériault et à Martin Meunier, qui signent conjointement un texte au titre volontairement provocateur et intitulé «Que reste-t-il de l'intention vitale du Canada français "? Au premier abord, ce texte se présente 
comme une critique de ce que les auteurs nomment «l'École de Toronto», une école de pensée dont les principaux animateurs (Monica Heller et Normand Labrie) se sont évertués depuis plus d'une décennie à déconstruire le discours sur la nation canadienne-française. Pour l'école de Toronto, ce discours sur l'existence d'une nation canadienne-française non seulement ne correspondrait pas à la réalité sociologique (les francophones du Canada ont des origines diverses qui ne renvoient pas toutes à cette "prétendue» nation), mais aurait depuis toujours été mobilisée par une certaine élite francophone au Canada qui y aurait trouvé son intérêt «de classe» (p. 211). On l'aura deviné, la critique de Thériault et Meunier se fait cinglante. La théorie de l'école de Toronto vide de tout contenu substantiel l'existence des communautés francophones en Amérique, ce que les auteurs nomment son «intention vitale».

Cette erreur a une double conséquence. Conséquence, d’abord, au niveau de l'analyse: la théorie de l'École de Toronto ne permet pas de comprendre le parcours des francophonies canadiennes, à la fois leur histoire, mais aussi son actuelle volonté de déploiement. Pour Meunier et Thériault en effet, cette intention est toujours palpable dans la volonté des communautés francophones de faire l'Amérique autrement, avec ses propres écoles, centres culturels, son propre réseau de santé, et ainsi de suite. En d'autres termes, les auteurs reprochent tout simplement à cette théorie d'être fausse. Autre conséquence, plus grave cette fois, les théories de cette école contribuent à un mouvement d'ethnicisation des francophones du Canada. Ethnicisation au sens où les francophones du Canada sont perçus (et se perçoivent) de moins en moins comme formant une nation, et de plus en plus comme un agrégat d'individus ayant fait le choix de parler français (on se demande bien pourquoi, d'ailleurs). Ces individus auraient des droits en vertu de ce choix, plutôt que comme des membres d'une communauté partageant un même projet, une même intention. Les théories, comme celle de l'école de Toronto, appuient scientifiquement les raisons fortes de cette ethnicisation. Cela a un impact, par exemple en salle de classe, lorsqu'on délaisse l'enseignement de l'existence de ce peuple, au profit d'une compréhension individualiste de la présence de francophones au Canada. On sent, à travers la critique de l'École de Toronto, que c'est une critique d'un mouvement plus large que déplorent les deux auteurs.

La dernière partie de l'ouvrage compte quatre textes qui s'intéressent à la politique et au droit. Linda Cardinal s'interroge sur le déficit démocratique dont souffrent les francophones du Canada vivant en situation 
minoritaire. S'ils aspirent à maîtriser leur destin, l'absence de frontières géographiques bien définies en limite les possibilités par la voie politique traditionnelle. En d'autres termes, les francophones du Canada, même dans les provinces où ils forment un fort contingent de la population (comme au Nouveau-Brunswick) n'ont pas de gouvernement en propre. Le milieu associatif constitue une forme de palliatif à ce problème, mais ce palliatif reste foncièrement insatisfaisant puisque les associations ne sont que très peu redevables démocratiquement devant les membres de ces communautés. Cardinal présente une excellente synthèse des voies (et surtout limites) des formes d'autogouvernement des francophones du Canada dans les domaines constitutionnel, administratif et électoral. Elle conclut à un accroissement de ce déficit démocratique depuis l'adoption de la Charte des droits et libertés. De manière paradoxale, la judiciarisation des questions identitaires qui a suivi l'adoption de la Charte a permis des gains importants pour les communautés francophones au niveau de la reconnaissance de leurs droits, mais a aussi présidé à une bureaucratisation accélérée du milieu associatif francophone, davantage au diapason des orientations gouvernementales que des aspirations démocratiques des communautés francophones.

Le texte de Johanne Poirier fait écho aux préoccupations de Cardinal. Pour l'auteure, le fédéralisme, comme régime politique, reflète peu la dualité linguistique canadienne, et a été largement défavorable aux communautés francophones du Canada qui n'a pas une véritable voix, même sur des sujets qui les touchent directement. Anne-Andrée Denault tente pour sa part de démystifier la question des rapports troubles entre la francophonie canadienne et le Québec. Elle montre, de manière convaincante, que la politique du gouvernement du Québec en matière de francophonie canadienne a été plutôt soutenue depuis la Révolution tranquille, contrairement au mythe - tenace - du total désintérêt des divers gouvernements face au sort de la francophonie canadienne.

Enfin, le texte de Pierre Foucher fait un exposé des principales victoires - mais aussi de quelques revers - enregistrées devant les tribunaux par les francophones du Canada depuis l'adoption de la Charte canadienne des droits et libertés.

Il est sans doute cliché de qualifier un ouvrage collectif d'«inégal». Même si le livre compte des textes moins percutants, il échappe largement à ce qualificatif tant la grande majorité des textes apportent un éclairage fouillé et intéressant sur la réalité des francophones du Canada. Concluons donc par un autre cliché: cet ouvrage deviendra rapidement un incon- 
tournable pour tous ceux qui s'intéressent à l'espace francophone en milieu minoritaire au Canada.

FRANÇOIS CHARBONNEAU

École d'études politiques

Université d'Ottawa

TULCHINSKY, Gerald, Canada's Jews, A People's Journey (Toronto, University of Toronto Press, 2008), $630 \mathrm{p}$.

Depuis une période récente, nous assistons à un foisonnement d'études sur la communauté juive au Canada qui prennent la forme de mémoires, de thèses, d'articles, d'essais et de monographies. Il convenait depuis un certain temps de rassembler ces études, souvent peu accessibles au grand public, tant par leur forme que par leur contenu, en une vaste synthèse. Gerald Tulchinsky avait déjà défriché ce champ d'études dans deux volumes, Taking Root et Branching Out, qui totalisaient plus de huit cents pages. Une étude volumineuse qui pouvait rebuter bon nombre de lecteurs. Canada's Jews, A People's Journey est donc une version remaniée des deux volumes précédents. Loin d'être un mauvais collage, l'auteur réussit à nous offrir une version qui conserve l'essentiel de l'information ainsi que l'unité et la cohérence du récit qui rendent la lecture facile et agréable à tous les lecteurs.

Cet ouvrage porte bien son titre. Il s'agit véritablement d'un voyage au cœur de la communauté juive au Canada pendant deux siècles d'histoire. La thèse de l'ouvrage vise à démontrer comment la culture et l'identité des immigrants juifs ont été influencées par le contexte de leur société d'accueil. D'après l'auteur, ce sont les conditions propres au Canada qui ont donné à la communauté juive ce caractère unique qui la distingue des communautés juives d'Europe et surtout des États-Unis. Pour ce faire, Tulchinsky reconstitue l'univers religieux, culturel, idéologique, social, économique et politique de la communauté juive au Canada de la fin du $\mathrm{XVIII}^{\mathrm{e}}$ siècle à aujourd'hui. Il reprend bien sûr des thèmes connus - pour ne pas dire "classiques» - comme les élites sociales et économiques, l'antisémitisme, les politiques d'immigration du gouvernement fédéral, le mouvement ouvrier, la question scolaire au Québec, etc. Toutefois, il sort des sentiers battus en s'intéressant aux tentatives d'implantation de colonies juives dans les prairies, à la personnalité anglo-canadienne antisémite de Goldwin Smith - moins connu du public francophone -, au 